\title{
Physico-Chemical Studies of Reactions of Alkalytion of Schiff's Bases
}

\author{
Mowafaq Y. Shandala Abdul-Wahab J. Al-Hamdany \\ Zaynab W.Majeed Omar A.Shareef \\ Department of Chemistry / College of Sciences \\ University of Mousl
}

Received

17 / 03 / 2009
Accepted

05 / 05 / 2009

\section{الخلصة:}

تمت الكلة قواعدشيف (N -ارايليين بنزايل لمين 1 -29) مع هاليدات الكيل متنوعة

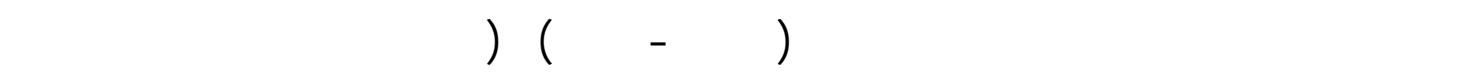

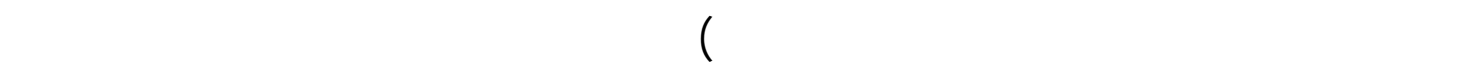

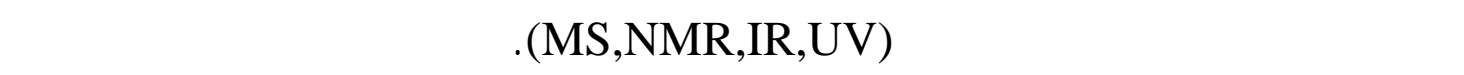
يمر عبرها الفاعل اعتمادا على المقاربة الظرية بلستعمل موديل AM1 بشبه الحرج) في برنلمج aن نوع ChemOffice الطراز الجزيئي.

\section{Abstract}

The Schiff's Bases (N-arylidene benzylamines) (1-29) have been alkylated with different alkyl halides by applying solid-liquid phasetransfer catalysis system (solid $\mathrm{K}_{2} \mathrm{CO}_{3}, \mathrm{CH}_{3} \mathrm{CN}$, TBAB). The products were identified as the corresponding alkylated Schiff's bases, by analyses of their spectral data (UV, IR, NMR, MS). The mechanism of the reaction was investigated on the basis of theoretical approach using semiempirical AM1 module in the CS ChemOffice molecular modeling package.

Keywords: Schiffs bases,Alkylation, N-Arylidene benzylamine.

\section{Introduction:}

Phase-transfer catalysis (PTC) technique had been used in the beginning $^{(1)}$ of the sixties as a new method to overcome the difficulties met in some reactions within immiscible compounds (heterogeneous 
solution). As an example of these difficulties, consider $^{(2)}$ the following reaction:

$$
\mathrm{n}-\mathrm{C}_{8} \mathrm{H}_{17} \mathrm{Cl}+\mathrm{NaCN} \stackrel{\mathrm{H}_{2} \mathrm{O}}{\longrightarrow} \mathrm{NaCl}+\mathrm{n}^{-} \mathrm{C}_{8} \mathrm{H}_{17} \mathrm{CN}
$$

This reaction will not proceed even after a continuous stirring for several days. To solve this problem, some special solvents have been used which have polar and non-polar sites such as: DMSO, DMF. These solvents are $a_{b l e}^{(3)}$ to dissolve the polar $(\mathrm{NaCN})$ and non-polar $\left(\mathrm{n}-\mathrm{C}_{8} \mathrm{H}_{17} \mathrm{Cl}\right)$. They also have some disadvantages ${ }^{(4)}$, like their high boiling points and being expensive and difficult to purify. It has been noticed that the reaction (below) is followed easily with good yield and rate when using a salt such as tetrabutyl ammonium cyanide ${ }^{(2)}$ instead of insoluble sodium cyanide:

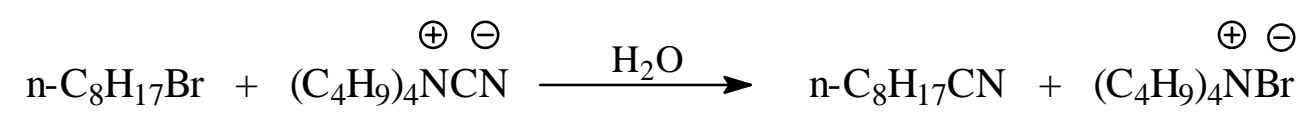

This is related to the lipophilic quaternary cation $\left[\mathrm{Q}^{+}=\left(\mathrm{C}_{4} \mathrm{H}_{9}\right)_{4} \mathrm{~N}^{+}\right]$ which transfers the cyanide ion to the organic phase as $\left(\mathrm{Q}^{+} \mathrm{CN}^{-}\right)$, and which in turn undergoes a reaction with $\left(\mathrm{n}-\mathrm{C}_{8} \mathrm{H}_{17} \mathrm{Br}\right)$ to yield cyanootcane and $\left(\mathrm{Q}^{+} \mathrm{Br}{ }^{-}\right)$which is quickly changed into $\left(\mathrm{Q}^{+} \mathrm{CN}^{-}\right)$either in the aqueous phase or in the interfacial surface as it is shown below:

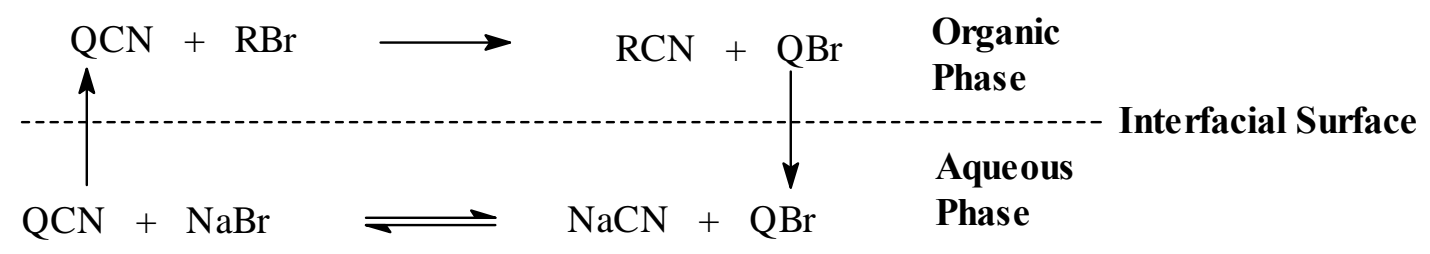

For the control of infectious diseases, the discovery and development of antibiotics are aiming the most powerful and successful achievements to modern science an technology ${ }^{(5)}$.

In the field of Schiff bases an extension investigations have been reported $^{(6,7)}$.

Chemical and Physical properties as well as their preparation have been described by various workers ${ }^{(8,9)}$. Many Workers denoted that Schiff bases formed from aromatic aldehydes or aromatic ketones and their substituents are quite stable. A wide range of these compounds have been synthesized due to the great diverse and flexibility in the structural aspects of Schiff bases, their complexation behavior had also been studied ${ }^{(10,11)}$. It was reported that nitro and halo derivatives of Schiff bases have antimicrobial and antitumor activites ${ }^{(12)}$. Antifugal and antimicrobial activates of various Schiff bases have also been reported $^{(13,14,15)}$. Sahu et al ${ }^{(16)}$. reported fungi toxicity of some Schiff bases. Gawad et $\mathrm{al}^{(17)}$. Synthesized some Schiff bases and observed high antimicrobial activites. Many Schiff bases are known to be medicinally important and are used to design medicinal compounds ${ }^{(18-20)}$. 


\section{Instrumentation}

\section{Experimental}

I. Boiling points were determined by inverted capillary in a Thiele tube using paraffin ${ }^{(21)}$ colourless oil.

II. Ultra-violet spectra were obtained using Shimadzu UV-Vissible spectrophotometer UV-160.

III. Infra-red spectra were recorded on Perkin-Elmer 590 B spectrophotometer.

IV. a). Nuclear magnetic resonance $\left({ }^{1} \mathrm{H}-\mathrm{NMR}\right)$ spectra were registered at $60 \mathrm{MHz}$. Hitachi Perkin-Elmer spectrometer, using tetramethyl silane (TMS) as an internal standard, and $\mathrm{CDCl}_{3}$, $\mathrm{d}_{6}$-DMSO as solvents.

b). Some compounds had been analysed in France* by Brucker AC 300, $400 \mathrm{MHz}{ }^{1} \mathrm{H}-\mathrm{NMR}$ spectrometer, using $\mathrm{CDCl}_{3}$ as a solvent.

The following abbreviations used to discuss the NMR data: $\mathrm{s}$ (singlet), d(doublet), 2d(two doublet signals), t(triplet), q(quartet), $\mathrm{p}$ (pentet), m(multiplet), br(broad).

V. Mass spectra (MS) were recorded in the laboratories of:

*UNIVERSITE`RENE`DESCARTES-PARIS V

DEPARTEMENT DE PHARMACOGNOSIE

FACULTE` DES SCIENCES PHARMACEU TIQUES ET BIOLOGIQUES

VI. Theoritical calculations based on the data obtained from the minimized geometery were computed using semi-empirical $\mathrm{AM}_{1}$ module in the CS ChemOffice molecular modeling package.

VII. The preliminary biological study was carried out in Dept. of Biology, Coll. of Sci., Univ. of Mosul. The standard Kirby and Bauer method was used.

\section{Alkylation of Schiff Bases Under (PTC) Conditions Using (Solid- Liquid) System \\ General Procedure ${ }^{(22)}$}

In a $100 \mathrm{ml}$ round-bottomed flask, a heterocyclic mixture of: Schiff base (10) mmole, catalyst (1) mmole, potassium carbonate (4 gm, 30 mmole), the corresponding electrophile (RX) (15) mmole and acetonitrile $\mathrm{CH}_{3} \mathrm{CN}$ (30) ml was stirred at room temperature until no further change in colour. The resulting mixture was filtered, and the solvent was evaporated under vacuum. The liquid product was then distilled. The products were analyzed.

Table (1) illustrate the spectral data (U.V and I.R), elemental data and some physical properties of the alkylated Schiff bases (1-20), whereas table (2) illustrates the amounts of alkyl halides along with the names of products (1-29). 
Table (1): Spectral and elemental data of alkylated Schiff bases (1-20).

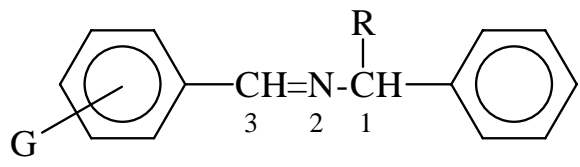

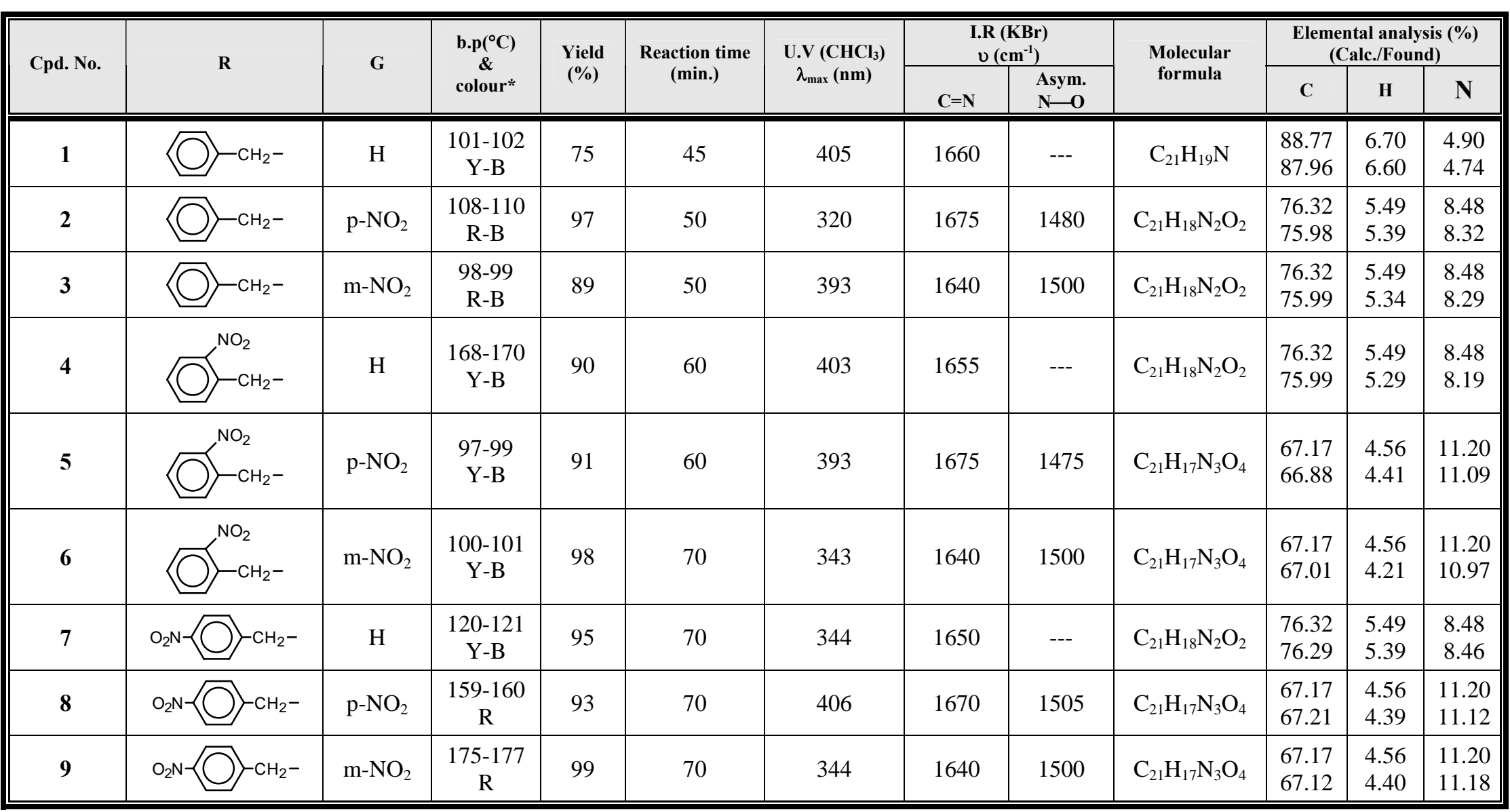

* Y: Yellow, R: Red, O: Orange, Y-B: Yellow-Brown, R-B: Reddish-Brown, P-B: Pale-Brown, D-B: Dark-Brown

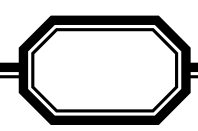


Table (1): Continued

\begin{tabular}{|c|c|c|c|c|c|c|c|c|c|c|c|c|}
\hline 10 & $\mathrm{CH}_{3} \mathrm{CH}=\mathrm{CHCH}_{2^{-}}$ & $\mathrm{H}$ & $\begin{array}{c}123-124 \\
\text { P-B }\end{array}$ & 97 & 30 & 343 & 1650 & --- & $\mathrm{C}_{18} \mathrm{H}_{17} \mathrm{NO}$ & $\begin{array}{l}87.39 \\
86.98\end{array}$ & $\begin{array}{l}6.93 \\
7.06\end{array}$ & $\begin{array}{l}5.66 \\
5.49\end{array}$ \\
\hline 11 & $\mathrm{CH}_{3} \mathrm{CH}=\mathrm{CHCH}_{2^{-}}$ & $\mathrm{p}-\mathrm{NO}_{2}$ & $\begin{array}{l}\text { 136-139 } \\
\text { D-B }\end{array}$ & 91 & 30 & 344 & 1650 & 1500 & $\mathrm{C}_{18} \mathrm{H}_{16} \mathrm{~N}_{2} \mathrm{O}_{3}$ & $\begin{array}{l}73.55 \\
73.46\end{array}$ & $\begin{array}{l}8.03 \\
8.10\end{array}$ & $\begin{array}{l}8.85 \\
8.79\end{array}$ \\
\hline 12 & $\mathrm{CH}_{3} \mathrm{CH}=\mathrm{CHCH}_{2^{-}}$ & $\mathrm{m}-\mathrm{NO}_{2}$ & $\begin{array}{c}131.5-132 \\
\text { P-B }\end{array}$ & 99 & 35 & 344 & 1640 & 1495 & $\mathrm{C}_{18} \mathrm{H}_{16} \mathrm{~N}_{2} \mathrm{O}_{3}$ & $\begin{array}{l}73.55 \\
73.50 \\
\end{array}$ & $\begin{array}{l}8.03 \\
8.00 \\
\end{array}$ & $\begin{array}{l}8.85 \\
8.32 \\
\end{array}$ \\
\hline 13 & $\mathrm{CH}_{2}=\mathrm{CHCH}_{2^{-}}$ & $\mathrm{p}-\mathrm{NO}_{2}$ & $\begin{array}{c}128-130 \\
\mathrm{R}\end{array}$ & 96 & 25 & 404 & 1640 & 1500 & $\mathrm{C}_{17} \mathrm{H}_{16} \mathrm{~N}_{2} \mathrm{O}_{2}$ & $\begin{array}{l}72.82 \\
72.76\end{array}$ & $\begin{array}{l}5.75 \\
5.69\end{array}$ & $\begin{array}{l}9.99 \\
9.82\end{array}$ \\
\hline 14 & $\mathrm{CH}_{2}=\mathrm{CHCH}_{2-}$ & $\mathrm{m}-\mathrm{NO}_{2}$ & $\begin{array}{c}88-90 \\
\text { B }\end{array}$ & 98 & 25 & 373 & 1650 & 1500 & $\mathrm{C}_{17} \mathrm{H}_{16} \mathrm{~N}_{2} \mathrm{O}_{2}$ & $\begin{array}{l}72.82 \\
72.70\end{array}$ & $\begin{array}{l}5.75 \\
5.66\end{array}$ & $\begin{array}{l}9.99 \\
9.82\end{array}$ \\
\hline 15 & & $\mathrm{H}$ & $\begin{array}{c}99-99.5 \\
\text { B }\end{array}$ & 96 & 20 & 344 & 1650 & --- & $\mathrm{C}_{22} \mathrm{H}_{17} \mathrm{NO}_{2}$ & $\begin{array}{l}89.44 \\
89.32\end{array}$ & $\begin{array}{l}5.80 \\
5.69\end{array}$ & $\begin{array}{l}4.74 \\
4.62\end{array}$ \\
\hline 16 & & $\mathrm{p}-\mathrm{NO}_{2}$ & $\begin{array}{c}\text { 119-120 } \\
\text { D-R }\end{array}$ & 98 & 20 & 408 & 1675 & 1480 & $\mathrm{C}_{22} \mathrm{H}_{16} \mathrm{~N}_{2} \mathrm{O}_{4}$ & $\begin{array}{l}70.94 \\
70.69\end{array}$ & $\begin{array}{l}4.33 \\
4.24\end{array}$ & $\begin{array}{l}7.52 \\
7.41\end{array}$ \\
\hline 17 & & $\mathrm{~m}-\mathrm{NO}_{2}$ & $\begin{array}{c}92-94 \\
\text { B }\end{array}$ & 98 & 25 & 343 & 1680 & 1450 & $\mathrm{C}_{22} \mathrm{H}_{16} \mathrm{~N}_{2} \mathrm{O}_{4}$ & $\begin{array}{l}70.94 \\
70.72\end{array}$ & $\begin{array}{l}4.33 \\
4.28\end{array}$ & $\begin{array}{l}7.52 \\
7.44\end{array}$ \\
\hline 18 & $\mathrm{Br}-\left(\mathrm{CH}_{2}\right)_{3}{ }^{-} \mathrm{CH}_{2}-$ & $\mathrm{H}$ & $\begin{array}{c}101-102 \\
Y\end{array}$ & 96 & 45 & 339 & 1650 & --- & $\mathrm{C}_{18} \mathrm{H}_{20} \mathrm{BrN}$ & $\begin{array}{l}62.21 \\
62.60\end{array}$ & $\begin{array}{l}5.80 \\
5.73\end{array}$ & $\begin{array}{l}4.03 \\
4.10\end{array}$ \\
\hline 19 & $\mathrm{Br}-\left(\mathrm{CH}_{2}\right)_{3^{-}}-\mathrm{CH}_{2}-$ & $\mathrm{p}-\mathrm{NO}_{2}$ & $\begin{array}{c}98-99 \\
\mathrm{O}\end{array}$ & 98 & 45 & 381 & 1650 & 1490 & $\mathrm{C}_{18} \mathrm{H}_{19} \mathrm{BrN}_{2} \mathrm{O}_{2}$ & $\begin{array}{l}57.59 \\
57.34 \\
\end{array}$ & $\begin{array}{l}5.10 \\
5.21\end{array}$ & $\begin{array}{l}7.46 \\
7.13 \\
\end{array}$ \\
\hline 20 & $\mathrm{Br}-\left(\mathrm{CH}_{2}\right)_{3}-\mathrm{CH}_{2}-$ & $\mathrm{m}-\mathrm{NO}_{2}$ & $\begin{array}{c}95-97 \\
\text { P-B } \\
\end{array}$ & 99 & 50 & 344 & 1675 & 1475 & $\mathrm{C}_{18} \mathrm{H}_{19} \mathrm{BrN}_{2} \mathrm{O}_{2}$ & $\begin{array}{l}57.59 \\
57.22 \\
\end{array}$ & $\begin{array}{l}5.10 \\
4.98 \\
\end{array}$ & $\begin{array}{l}7.46 \\
7.22 \\
\end{array}$ \\
\hline
\end{tabular}

* Y: Yellow, R: Red, O: Orange, Y-B: Yellow-Brown, R-B: Reddish-Brown, P-B: Pale-Brown, D-B: Dark-Brown 
Table (2): Names of alkylated Schiff bases (1-29)

\begin{tabular}{|c|c|c|c|c|c|}
\hline $\begin{array}{l}\text { Cpd } \\
\text {.No. }\end{array}$ & $\begin{array}{c}\text { Alkyl halide } \\
\text { (Name \& amount) }\end{array}$ & Name of alkylated Schiff base & $\begin{array}{l}\text { Cpd } \\
\text {. No. }\end{array}$ & $\begin{array}{l}\text { Alkyl halide } \\
\text { (Name \& amount) }\end{array}$ & Name of alkylated Schiff base \\
\hline 1 & \multirow{3}{*}{$\begin{array}{l}\text { Benzyl chloride } \\
\quad(1.72) \mathrm{ml}\end{array}$} & N-Benzylidene- $\alpha$-benzyl benzyl amine & 15 & \multirow{3}{*}{$\begin{array}{c}\text { Methyl-3,4- } \\
\text { dioxobenzyl chloride } \\
\text { (2.4) gm }\end{array}$} & $\begin{array}{l}\text { N-Benzylidene- } \alpha \text {-(3,4-dioxymethyl } \\
\text { benzyl) benzyl amine }\end{array}$ \\
\hline 2 & & $\begin{array}{l}\text { N-(p-Nitrobenzylidene)- } \alpha \text {-benzyl benzyl } \\
\text { amine }\end{array}$ & 16 & & $\begin{array}{l}\mathrm{N} \text {-(p-Nitrobenzylidene)- } \alpha \text {-(3,4-dioxy } \\
\text { methyl benzyl) benzyl amine }\end{array}$ \\
\hline 3 & & $\begin{array}{l}\text { N-(m-Nitrobenzylidene)- } \alpha \text {-benzyl benzyl } \\
\text { amine }\end{array}$ & 17 & & $\begin{array}{l}\text { N-(m-Nitrobenzylidene)- } \alpha \text {-(3,4-dioxy } \\
\text { methyl benzyl) benzyl amine }\end{array}$ \\
\hline 4 & \multirow{3}{*}{$\begin{array}{l}\text { o-Nitrobenzyl } \\
\text { chloride } \\
\text { (2.56) gm }\end{array}$} & $\begin{array}{l}\text { N-Benzylidene- } \alpha \text {-(o-nitrobenzyl) benzyl } \\
\text { amine }\end{array}$ & 18 & \multirow{3}{*}{$\begin{array}{l}\text { 1,4-Dibromo butane } \\
\text { (1.79) } \mathrm{ml}\end{array}$} & $\begin{array}{l}\text { N-Benzylidene-1-phenyl-5-bromo pentyl } \\
\text { amine }\end{array}$ \\
\hline 5 & & $\begin{array}{l}\mathrm{N} \text {-(p-Nitrobenzylidene)- } \alpha \text {-(o-nitro- benzyl) } \\
\text { benzyl amine }\end{array}$ & 19 & & $\begin{array}{l}\text { N-(p-Nitrobenzylidene)-1-phenyl-5-bromo } \\
\text { pentyl amine }\end{array}$ \\
\hline 6 & & $\begin{array}{l}\mathrm{N} \text {-(m-Nitrobenzylidene)- } \alpha \text {-(o-nitro-benzyl) } \\
\text { benzyl amine }\end{array}$ & 20 & & $\begin{array}{l}\text { N-(m-Nitrobenzylidene)-1-phenyl-5- } \\
\text { bromo pentyl amine }\end{array}$ \\
\hline 7 & \multirow{2}{*}{$\begin{array}{l}\text { p-Nitrobenzyl } \\
\text { chloride } \\
(2.56)\end{array}$} & $\begin{array}{l}\text { N-Benzylidene- } \alpha \text {-(p-nitrobenzyl) benzyl } \\
\text { amine }\end{array}$ & 21 & \multirow[b]{2}{*}{$\begin{array}{l}\text { Methyl iodide } \\
\text { (0.93) ml }\end{array}$} & N-Benzylidene- $\alpha$-methyl benzyl amine \\
\hline 8 & & $\begin{array}{l}\mathrm{N}-(\mathrm{p}-\mathrm{Nitrobenzylidene})-\alpha-(\mathrm{p}-\text { nitro- benzyl) } \\
\text { benzyl amine }\end{array}$ & 22 & & $\begin{array}{l}\text { N-(p-Nitrobenzylidene)- } \alpha \text {-methyl benzyl } \\
\text { amine }\end{array}$ \\
\hline 10 & \multirow{3}{*}{$\begin{array}{l}\text { Crotyl chloride } \\
\quad(1.47) \mathrm{ml}\end{array}$} & N-Benzylidene-1-phenyl-3-pentenyl amine & 24 & \multirow{3}{*}{$\begin{array}{l}\text { Butyl bromide } \\
\text { (1.6) } \mathrm{ml}\end{array}$} & N-Benzylidene-1-phenyl pentyl amine \\
\hline 11 & & $\begin{array}{l}\text { N-(p-Nitrobenzylidene)- 1-phenyl-3- } \\
\text { pentenyl amine }\end{array}$ & 25 & & $\begin{array}{l}\text { N-(p-Nitrobenzylidene)-1-phenyl pentyl } \\
\text { amine }\end{array}$ \\
\hline 12 & & $\begin{array}{l}\text { N-(m-Nitrobenzylidene)-1-phenyl-3- } \\
\text { pentenyl amine }\end{array}$ & 26 & & $\begin{array}{l}\text { N-(m-Nitrobenzylidene)-1-phenyl pentyl } \\
\text { amine }\end{array}$ \\
\hline 13 & \multirow{3}{*}{$\begin{array}{l}\text { Allyl chloride } \\
\quad(1.22) \mathrm{ml}\end{array}$} & $\begin{array}{l}\text { N-(p-Nitrobenzylidene)-1-phenyl-3-butenyl } \\
\text { amine }\end{array}$ & 27 & \multirow{3}{*}{$\begin{array}{l}\text { o-Bromobenzoyl } \\
\text { chloride } \\
\text { (2.19) } \mathrm{ml}\end{array}$} & $\begin{array}{l}\text { N-Benzylidene- } \alpha \text {-(o-bromobenzoyl)- } \\
\text { benzyl amine }\end{array}$ \\
\hline \multirow[t]{2}{*}{14} & & $\begin{array}{l}\text { N-(m-Nitrobenzylidene)-1-phenyl-3- } \\
\text { butenyl amine }\end{array}$ & 28 & & $\begin{array}{l}\mathrm{N} \text {-(p-Nitrobenzylidene)- } \alpha \text {-(o-bromo } \\
\text { benzoyl) benzyl amine }\end{array}$ \\
\hline & & & 29 & & $\begin{array}{l}\mathrm{N} \text {-(m-Nitrobenzylidene)- } \alpha \text {-(o-bromo } \\
\text { benzoyl) benzyl amine }\end{array}$ \\
\hline
\end{tabular}


Table (3): Spectral data, physical properties and elemental data of some alkylated Schiff bases(21-29).

\begin{tabular}{|c|c|c|c|c|c|c|c|c|c|c|c|c|c|}
\hline \multirow[t]{2}{*}{$\begin{array}{c}\text { Cpd. } \\
\text { No. }\end{array}$} & \multirow[t]{2}{*}{$\begin{array}{c}\text { b.p }\left({ }^{\circ} \mathrm{C}\right) \\
\& \\
\text { colour* }\end{array}$} & \multirow[t]{2}{*}{$\mathbf{G}$} & \multirow[t]{2}{*}{$\mathbf{R}$} & \multirow{2}{*}{$\begin{array}{c}\mathbf{U V} \\
\left(\mathrm{CHCl}_{3}\right) \\
\lambda \max \\
(\mathbf{n m})\end{array}$} & \multicolumn{2}{|c|}{$\begin{array}{l}\operatorname{IR}(\mathrm{KBr}) \\
U\left(\mathrm{~cm}^{-1}\right)\end{array}$} & \multirow{2}{*}{$\begin{array}{c}{ }^{1} \mathrm{H}-\mathrm{NMR}\left(\mathrm{CDCl}_{3}\right) \\
\delta(\mathrm{ppm})\end{array}$} & \multirow{2}{*}{$\begin{array}{l}\text { Yield } \\
(\%)\end{array}$} & \multirow{2}{*}{$\begin{array}{l}\text { Reactio } \\
\text { n time } \\
\text { (min) }\end{array}$} & \multirow{2}{*}{$\begin{array}{l}\text { Molecula } \\
\text { r formula }\end{array}$} & \multicolumn{3}{|c|}{$\begin{array}{c}\text { Elemental analysis } \\
(\%) \\
\text { (Calc./Found)) } \\
\end{array}$} \\
\hline & & & & & $\mathrm{C}=\mathrm{N}$ & $\begin{array}{l}\mathrm{N}-\mathrm{O} \\
\text { asym }\end{array}$ & & & & & $\mathbf{C}$ & $\mathbf{H}$ & $\mathbf{N}$ \\
\hline 21 & $\begin{array}{c}94-97 \\
Y-B\end{array}$ & $\mathrm{H}$ & $\mathrm{CH}_{3}-$ & 304 & 1650 & --- & $\begin{array}{l}0.9\left(\mathrm{~d}, 3 \mathrm{H}, \mathrm{CH}_{3}\right), 3.0(\mathrm{q}, \\
1 \mathrm{H}, \mathrm{H}-1), 5.55(\mathrm{~s}, 1 \mathrm{H}, \\
\mathrm{H}-3), 6.9(\mathrm{~s}, 10 \mathrm{H}, \mathrm{Ar}- \\
\mathrm{H})\end{array}$ & 88 & 15 & $\mathrm{C}_{15} \mathrm{H}_{15} \mathrm{~N}$ & $\begin{array}{l}86.07 \\
86.17\end{array}$ & $\begin{array}{l}7.22 \\
7.01\end{array}$ & $\begin{array}{l}6.69 \\
6.42\end{array}$ \\
\hline 23 & $\begin{array}{c}87-88 \\
Y-B\end{array}$ & $\mathrm{~m}-\mathrm{NO}_{2}$ & $\mathrm{CH}_{3^{-}}$ & 302 & 1640 & 1500 & $\begin{array}{l}\left.\text { 1.0(m,3H, } \mathrm{CH}_{3}\right), \\
4.35(\mathrm{q}, 1 \mathrm{H}, \mathrm{H}-1), \\
5.5(\mathrm{~s}, 1 \mathrm{H}, \mathrm{H}-3), 6.8-7.5 \\
(\mathrm{~m}, 9 \mathrm{H}, \mathrm{Ar}-\mathrm{H})\end{array}$ & 87 & 15 & $\begin{array}{c}\mathrm{C}_{15} \mathrm{H}_{14} \mathrm{~N}_{2} \mathrm{O} \\
2\end{array}$ & $\begin{array}{l}70.83 \\
70.57\end{array}$ & $\begin{array}{l}5.55 \\
5.38\end{array}$ & $\begin{array}{l}11.02 \\
11.20\end{array}$ \\
\hline
\end{tabular}


Table (3): Continued

\begin{tabular}{|c|c|c|c|c|c|c|c|c|c|c|c|c|c|}
\hline 25 & $\begin{array}{c}\text { 164-165 } \\
\text { Y-B }\end{array}$ & $\mathrm{p}-\mathrm{NO}_{2}$ & $\mathrm{CH}_{3}\left(\mathrm{CH}_{2}\right)_{2} \mathrm{CH}_{2-}$ & 396 & 1640 & 1490 & $\begin{array}{l}0.9\left(\mathrm{t}, 3 \mathrm{H}, \mathrm{CH}_{3}\right), 1.4(\mathrm{~m}, 4 \mathrm{H}, \\
\left.-\mathrm{CH}_{2}-\mathrm{CH}_{2}\right), 1.7(\mathrm{~m}, 2 \mathrm{H}, \mathrm{Ar}- \\
\left.\mathrm{CH}_{2}\right), 3.0(\mathrm{t}, 1 \mathrm{H}, \mathrm{H}-1), 4.9 \\
(\mathrm{~m}, 1 \mathrm{H}, \mathrm{H}-3), 6.5-8.0(\mathrm{~m}, \\
\left.5 \mathrm{H}, \mathrm{C}_{6} \mathrm{H}_{5}-\right), 6.6-7.9(\mathrm{dd}, 4 \mathrm{H}, \\
\left.\text { p- } \mathrm{NO}_{26} \mathrm{H}_{4}\right)\end{array}$ & 92 & 40 & $\mathrm{C}_{18} \mathrm{H}_{20} \mathrm{~N}_{2} \mathrm{O}_{2}$ & $\begin{array}{l}81.76 \\
81.51\end{array}$ & $\begin{array}{l}7.63 \\
7.72\end{array}$ & $\begin{array}{l}10.60 \\
10.48\end{array}$ \\
\hline 26 & $\begin{array}{c}\text { 135-136 } \\
\text { Y-B }\end{array}$ & $\mathrm{m}-\mathrm{NO}_{2}$ & $\mathrm{CH}_{3}\left(\mathrm{CH}_{2}\right)_{2} \mathrm{CH}_{2^{-}}$ & 343 & 1640 & 1500 & $\begin{array}{l}0.9\left(\mathrm{t}, 3 \mathrm{H}, \mathrm{CH}_{3}\right), 1.5(\mathrm{~m}, 4 \mathrm{H}, \\
\left.-\mathrm{CH}_{2}-\mathrm{CH}_{2^{-}}\right), 2.0(\mathrm{~m}, 2 \mathrm{H}, \\
\left.\mathrm{Ar}-\mathrm{CH}_{2}-\right), 3.2(\mathrm{t}, 1 \mathrm{H}, \mathrm{H}-1) \\
5.0(\mathrm{~s}, 1 \mathrm{H}, \mathrm{H}-3), 6.5- \\
8.6(\mathrm{~m}, 9 \mathrm{H}, \mathrm{Ar}-\mathrm{H})\end{array}$ & 90 & 45 & $\mathrm{C}_{18} \mathrm{H}_{20} \mathrm{~N}_{2} \mathrm{O}_{2}$ & $\begin{array}{l}81.76 \\
81.62\end{array}$ & $\begin{array}{l}7.63 \\
7.45\end{array}$ & $\begin{array}{l}10.60 \\
10.62\end{array}$ \\
\hline 27 & $\begin{array}{c}89-90 \\
\text { B }\end{array}$ & $\mathrm{H}$ & & 300 & 1665 & --- & $\begin{array}{l}2.8(\mathrm{~s}, 1 \mathrm{H}, \mathrm{H}-1), 4.65(\mathrm{~s}, \\
1 \mathrm{H}, \mathrm{H}-3), 6.0-6.7(\mathrm{~m}, 10 \mathrm{H}, \\
\mathrm{Ar}-\mathrm{H}), 7.0-7.5(\mathrm{~m}, 4 \mathrm{H}, \mathrm{o}- \\
\left.\mathrm{Br}\left(6 \mathrm{H}_{4}\right)\right)\end{array}$ & 97 & 80 & $\mathrm{C}_{21} \mathrm{H}_{16} \mathrm{BrNO}$ & $\begin{array}{l}66.66 \\
66.41\end{array}$ & $\begin{array}{l}4.26 \\
4.19\end{array}$ & $\begin{array}{l}3.70 \\
3.60\end{array}$ \\
\hline 28 & $\begin{array}{c}136-137 \\
\text { D-R }\end{array}$ & $\mathrm{p}-\mathrm{NO}_{2}$ & & 399 & 1640 & 1465 & $\begin{array}{l}\text { 3.2(s, } 1 \mathrm{H}, \mathrm{H}-1), 5.6(\mathrm{~s}, 1 \mathrm{H}, \\
\mathrm{H}-3), 6.6-7.5(\mathrm{~m}, 9 \mathrm{H}, \mathrm{Ar}- \\
\mathrm{H}), 6.4-7.4(\mathrm{dd}, 4 \mathrm{H}, \mathrm{p}- \\
\left.\mathrm{NO}_{2} \mathrm{C}_{6} \mathrm{H}_{4^{-}}\right)\end{array}$ & 98 & 80 & $\begin{array}{c}\mathrm{C}_{21} \mathrm{H}_{15} \mathrm{BrN}_{2} \mathrm{O} \\
3\end{array}$ & $\begin{array}{l}59.55 \\
59.29\end{array}$ & $\begin{array}{l}3.57 \\
3.42\end{array}$ & $\begin{array}{l}6.61 \\
6.45\end{array}$ \\
\hline 29 & $\begin{array}{c}111-112 \\
\text { D-R }\end{array}$ & $\mathrm{m}-\mathrm{NO}_{2}$ & & 344 & 1650 & 1505 & $\begin{array}{l}3.3(\mathrm{~m}, 1 \mathrm{H}, \mathrm{H}-1), 5.3(\mathrm{~m}, \\
1 \mathrm{H}, \mathrm{H}-3), 6.2-8.0(\mathrm{~m}, 13 \mathrm{H}, \\
\text { Ar-H) }\end{array}$ & 98 & 90 & $\begin{array}{c}\mathrm{C}_{21} \mathrm{H}_{15} \mathrm{BrN}_{2} \mathrm{O} \\
3\end{array}$ & $\begin{array}{l}59.55 \\
59.28\end{array}$ & $\begin{array}{l}3.57 \\
3.42\end{array}$ & $\begin{array}{l}6.61 \\
6.50\end{array}$ \\
\hline
\end{tabular}


Table (4): The ${ }^{1} \mathrm{H}-\mathrm{NMR}$, Mass spectral and elemental data of compound (23)

\begin{tabular}{|c|c|c|c|c|c|c|c|c|c|c|c|}
\hline $\begin{array}{l}\text { Cdp. } \\
\text { No. }\end{array}$ & \multicolumn{2}{|c|}{$\begin{array}{c}\operatorname{NMR}\left(\mathrm{CDCl}_{3}\right) \\
\Delta(\mathrm{ppm})\end{array}$} & $\begin{array}{l}\text { Molecular } \\
\text { formula }\end{array}$ & \multicolumn{5}{|c|}{$\begin{array}{l}\text { Elemental Microanalysis \% } \\
\text { (Cal C. /Found) }\end{array}$} & \multicolumn{3}{|c|}{ Structure } \\
\hline & \multirow{2}{*}{\multicolumn{2}{|c|}{$\begin{array}{c}1.4\left(\mathrm{P}, 4 \mathrm{H}, \mathrm{CH}_{2}-3, \mathrm{CH}_{2}-\right. \\
4) \\
1.7\left(\mathrm{q}, 2 \mathrm{H}, \mathrm{CH}_{2}-2\right) \\
2.9\left(\mathrm{t}, \mathrm{CH}_{2}-31 \mathrm{H}, \mathrm{CH}-\right. \\
1) \\
3.55\left(5,2 \mathrm{H}, \mathrm{CH}_{2}-5\right) \\
8.4(5,1 \mathrm{H}, \mathrm{CH}=\mathrm{N}) \\
7.2-7.9(\mathrm{~m}, 10 \mathrm{H}, \mathrm{Ar}-\mathrm{H})\end{array}$}} & & C & $\mathbf{H}$ & Hal & $\mathbf{N}$ & $\mathbf{O}$ & & & \\
\hline 23 & & & $\mathrm{C}_{18} \mathrm{H}_{20} \mathrm{BrN}$ & $\begin{array}{l}65.43 \\
65.46\end{array}$ & $\begin{array}{l}6.10 \\
6.16\end{array}$ & $\begin{array}{l}24.20 \\
24.19\end{array}$ & $\begin{array}{l}4.24 \\
4.24\end{array}$ & $\begin{array}{l}- \\
--\end{array}$ & & & \\
\hline \multirow[b]{2}{*}{$\begin{array}{l}\text { Cdp. } \\
\text { No. }\end{array}$} & \multirow[b]{2}{*}{$\mathbf{m} / \mathbf{z}$} & \multirow{2}{*}{$\begin{array}{c}\text { Relative } \\
\text { intensity }(\%)\end{array}$} & \multirow{2}{*}{$\begin{array}{l}\text { Reagent } \\
\text { gas }\end{array}$} & \multirow{2}{*}{\multicolumn{2}{|c|}{$\begin{array}{l}\text { Integral }^{(27)} \\
\text { collector } \\
\text { reading } \\
\text { (abundance) }\end{array}$}} & \multirow{2}{*}{\multicolumn{3}{|c|}{$\begin{array}{c}\text { Molecular ion } \\
\text { and base } \\
\text { peak }^{(28)}\end{array}$}} & \multicolumn{3}{|c|}{ Parent molecule } \\
\hline & & & & & & & & & $\begin{array}{c}\text { Elemental } \\
\text { Composition }\end{array}$ & $\begin{array}{c}\text { Exact } \\
\text { mass }\end{array}$ & $\begin{array}{l}\text { Molecular } \\
\text { weight }\end{array}$ \\
\hline \multirow[b]{2}{*}{23} & 196 & 100 & \multirow[b]{2}{*}{$\mathbf{N H}_{3}$} & \multicolumn{2}{|c|}{11.400} & \multicolumn{3}{|c|}{ Base peak } & & & \\
\hline & 330 & 0.76 & & \multicolumn{2}{|c|}{100} & \multicolumn{3}{|c|}{$\begin{array}{c}\text { Molecular ion } \\
\mathbf{M H}^{+}\end{array}$} & $\mathrm{C}_{18} \mathrm{H}_{20} \mathrm{BrN}$ & 329.10 & 330.26 \\
\hline
\end{tabular}




\section{Resulst and Discussion}

The alkylation of Schiff bases [N-benzylidene benzylamine (I), N-(pnitrobenzylidene benzylamine) (II) and $\mathrm{N}$-(m-nitrobenzylidene benzylamine (III)] under (PTC) conditions (solid-liquid system) with different alkyl halides afforded the corresponding alkylated Schiff bases (1-29):

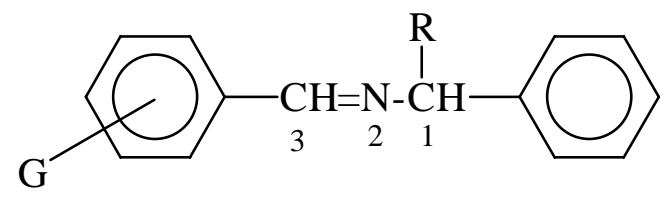

N-arylidene- $\alpha$-substituted benzylamines (1-29)

The spectral and elemental data are used for the identification of the products (1-29) (tables: 1, 2, 3 and 4).

\begin{tabular}{|c|c|c|c|c|c|}
\hline Cpd. No. & G & $\mathbf{R}$ & $\begin{array}{l}\text { Cpd. } \\
\text { No. }\end{array}$ & G & $\mathbf{R}$ \\
\hline 1 & $\mathrm{H}$ & $\mathrm{Ph}-\mathrm{CH}_{2-}$ & 15 & $\mathrm{H}$ & \\
\hline 2 & $\mathrm{p}-\mathrm{NO}_{2}$ & $=$ & 16 & $\mathrm{p}-\mathrm{NO}_{2}$ & $=$ \\
\hline 3 & $\mathrm{~m}-\mathrm{NO}_{2}$ & $=$ & 17 & $\mathrm{~m}-\mathrm{NO}_{2}$ & $=$ \\
\hline 4 & $\mathrm{H}$ & & 18 & $\mathrm{H}$ & $\mathrm{Br}\left(\mathrm{CH}_{2}\right)_{3} \mathrm{CH}_{2^{-}}$ \\
\hline 5 & $\mathrm{p}-\mathrm{NO}_{2}$ & $=$ & 19 & $\mathrm{p}-\mathrm{NO}_{2}$ & $=$ \\
\hline 6 & $\mathrm{~m}-\mathrm{NO}_{2}$ & $=$ & 20 & $\mathrm{~m}-\mathrm{NO}_{2}$ & $=$ \\
\hline 7 & $\mathrm{H}$ & $\mathrm{O}_{2} \mathrm{~N}-\mathrm{CH}_{2}$ & 21 & $\mathrm{H}$ & $\mathrm{CH}_{3^{-}}$ \\
\hline 8 & $\mathrm{p}-\mathrm{NO}_{2}$ & $=$ & 22 & $\mathrm{p}-\mathrm{NO}_{2}$ & $=$ \\
\hline 9 & $\mathrm{~m}-\mathrm{NO}_{2}$ & $=$ & 23 & $\mathrm{~m}-\mathrm{NO}_{2}$ & $=$ \\
\hline 10 & $\mathrm{H}$ & $\begin{array}{c}\mathrm{CH}_{3} \mathrm{CH}=\mathrm{CHC} \\
\mathrm{H}_{2^{-}}\end{array}$ & 24 & $\mathrm{H}$ & $\mathrm{CH}_{3}\left(\mathrm{CH}_{2}\right)_{2} \mathrm{CH}_{2}-$ \\
\hline 11 & $\mathrm{p}-\mathrm{NO}_{2}$ & $=$ & 25 & $\mathrm{p}-\mathrm{NO}_{2}$ & $=$ \\
\hline 12 & $\mathrm{~m}-\mathrm{NO}_{2}$ & $=$ & 26 & $\mathrm{~m}-\mathrm{NO}_{2}$ & $=$ \\
\hline 13 & $\mathrm{p}-\mathrm{NO}_{2}$ & $\mathrm{CH}_{2}=\mathrm{CHCH}_{2^{-}}$ & 27 & $\mathrm{H}$ & $\mathrm{Br}$ \\
\hline \multirow[t]{2}{*}{14} & $\mathrm{~m}-\mathrm{NO}_{2}$ & $=$ & 28 & $\mathrm{p}-\mathrm{NO}_{2}$ & $=$ \\
\hline & & & 29 & $\mathrm{~m}-\mathrm{NO}_{2}$ & $=$ \\
\hline
\end{tabular}

As a representative model, the compound (18) is selected in the discussion of spectral data.

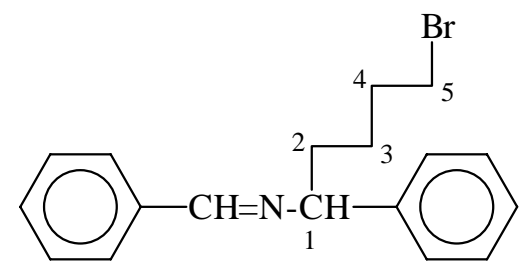


The $\left({ }^{1} \mathrm{H}-\mathrm{NMR}\right)$ spectrum ${ }^{(23,24)}$ (table 4) shows a pentet signal resonates at $\delta(1.4) \mathrm{ppm}(4 \mathrm{H})$ related to the methylene protons at $\mathrm{C}-3$ and C-4. A quartet signal at $\delta(1.7) \mathrm{ppm}(2 \mathrm{H})$ attributed to the methylene protons at C-2. A triplet signal resonates at $\delta(2.9) \mathrm{ppm}(1 \mathrm{H})$ corresponds to the proton at $\mathrm{C}-1$. A singlet signal is appeared at $\delta(3.55) \mathrm{ppm}(2 \mathrm{H})$ due to the methylene protons at $\mathrm{C}-5$. Another singlet signal resonates at $\delta(8.4)$ ppm $(1 \mathrm{H})$ referred to $\mathrm{C} \underline{\mathrm{H}}=\mathrm{N}$.

Finally, the multiplet signal at $\delta(7.2-7.9) \mathrm{ppm}(10 \mathrm{H})$ related to the aromatic protons.

The (IR) spectrum (tables 1) exhibits a strong absorption band at (1650) $\mathrm{cm}^{-1}$ attributed to $\mathrm{vC}=\mathrm{N}$.

The (UV) spectrum ${ }^{(25)}$ (table 1) indicates a maximum absorption at wavelength $\lambda \max =(339) \mathrm{nm}$ compared with $\lambda \max =(320) \mathrm{nm}$ for the reacted Schiff base (I) which reflects a red shift.

The (MS) spectrum ${ }^{(26,27)}$ (table 4) shows a base peak ${ }^{(28)}(100 \%$, abundance 11,400$)$ for $\mathrm{m} / \mathrm{z}=196$ and a molecular ion $(\mathrm{MH})^{+}(0.76 \%$, abundance 100) for $\mathrm{m} / \mathrm{z}=330$. The molecular ion $(\mathrm{MH})^{+}$is in quite good agreement with the composition $\mathrm{C}_{18} \mathrm{H}_{20} \mathrm{BrN}$.

The elemental analysis (table 4) shows a good agreement with the calculated $(\mathrm{C}, \mathrm{H}, \mathrm{N}, \mathrm{Br})$ values.

The alkylation of the Schiff base (N-benzylidene benzylamine I) using: benzyl chloride, crotyl chloride and methyl iodide are selected as a representative reactions for discussing the mechanism of the alkylation.

The suggested mechanism for such reaction (scheme 1) is initiated by the abstraction of the acidic proton from the Schiff base under the influence of the strong base $(50 \% \mathrm{NaOH})$. The formed anion $\mathrm{An}_{1}$ may attack the alkyl halide (RX) via nucleophilic substitution to afford the final products (1) $(\mathrm{H} . \mathrm{F}=93.98380, \mathrm{~S} . \mathrm{E}=0.206),(10)(\mathrm{H} . \mathrm{F}=75.28176$, $\mathrm{S} . \mathrm{E}=7.047)$ and $(21)(\mathrm{H} . \mathrm{F}=66.60970, \mathrm{~S} . \mathrm{E}=0.433)$.

$\mathrm{An}_{1}$ may resonates to give the anion $\mathrm{An}_{2}$ since $\mathrm{An}_{1}$ and $\mathrm{An}_{2}$ are the same, accordingly the same product is obtained whether the alkylation occurred at any one of the two carbons attached to the nitrogen, the mechanism may be suggested as the attack of the anion $\mathrm{An}_{1}$ or $\mathrm{An}_{2}$ on $\alpha$ carbon of R-X or via the resonance hybride $\mathrm{An}_{3}$.

Appendix (17) illustrates the minimized geometry data of the final product (21), whereas (Figure 1) illustrates the 3D-structure of (21). The $3 \mathrm{D}$-structure of (21) reflects the planarity of the molecule where:

$\mathrm{C}_{2} \mathrm{C}_{3} \mathrm{C}_{4} \underline{\mathrm{C}}_{5}=0.09484, \mathrm{C}_{3} \mathrm{C}_{2} \mathrm{C}_{1} \underline{\mathrm{C}}_{6}=0.05331, \mathrm{C}_{3} \mathrm{C}_{4} \mathrm{C}_{5} \underline{\mathrm{C}}_{7}=-179.75113$, $\mathrm{C}_{4} \mathrm{C}_{5} \mathrm{C}_{7} \mathrm{~N}_{8}=-179.22244, \quad \mathrm{C}_{5} \mathrm{C}_{7} \mathrm{~N}_{8} \quad \underline{\mathrm{C}}_{9}=179.28763$ and $\mathrm{C}_{9} \mathrm{C}_{10} \mathrm{C}_{11} \underline{\mathrm{C}}_{12}=$ -178.97775 (Appendix-1) 


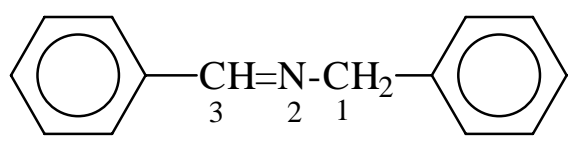

H.F. $=69.54028 \mathrm{Kcal} / \mathrm{mole}$

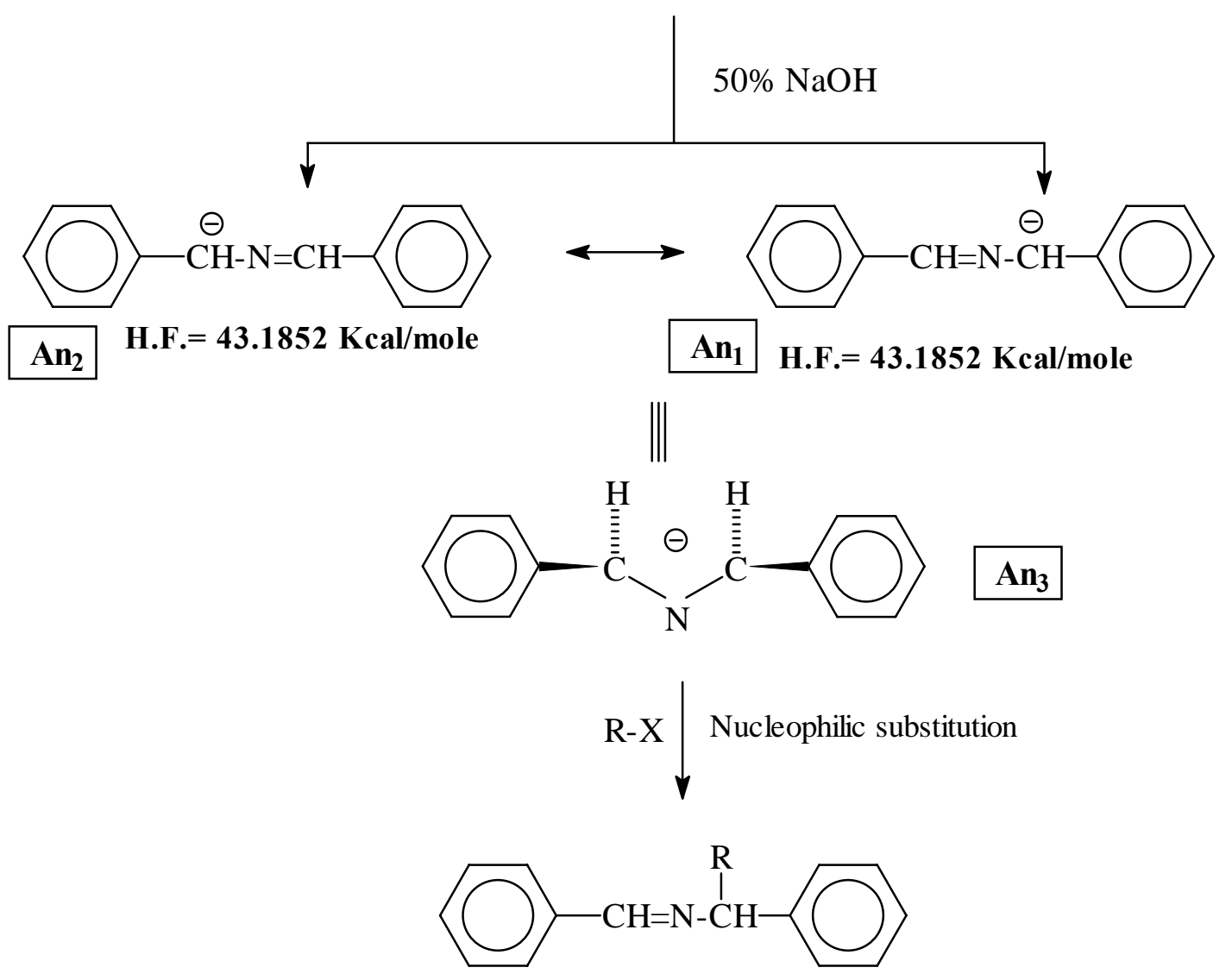

\begin{tabular}{|c|c|c|c|}
\hline Cpd. No. & $\underline{\mathrm{R}}=\underline{X}$ & 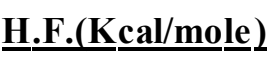 & S.E.(Kcal $/$ mole $)$ \\
\hline 1 & $-\mathrm{CH}_{2} \mathrm{Cl}$ & 93.98380 & 0.206 \\
\hline 10 & $\mathrm{CH}_{3} \mathrm{CH}=\mathrm{CHCH}_{2} \mathrm{Cl}$ & 75.28176 & 4.047 \\
\hline 21 & $\mathrm{CH}_{3} \mathrm{I}$ & 66.60970 & 0.433 \\
\hline
\end{tabular}

Scheme (1): Reaction of Schiff base (I) with benzyl chloride, crotyl chloride and methyl iodide 
On the other hand, when asymmetric Schiff base is used, i.e.: with certain substituents on one of the two phenyls as Schiff base (N-pnitrobenzylidene benzylamine), the attack may proceed via $\mathrm{An}_{1}$ or $\mathrm{An}_{2}$ (which may be represented by $\mathrm{An}_{3}$ ) on the alkyl halide.

The alkylation of Schiff base with benzyl chloride, crotyl chloride and methyl iodide which afforded (2, 11 and 22) respectively are investigated in order to give some information on the mechanism of these reactions and show the effect of the alkyl groups on such reactions.

As such, the abstraction of the acidic proton from the Schiff base may leads to the liberation of the two anions $\mathrm{An}_{1}$ and $\mathrm{An}_{2}$ (scheme 2) which may be represented by the resonance hybrid $\mathrm{An}_{3}$.

$\mathrm{An}_{3}$ in turn may attack the carbon attached to the halogen in the alkyl halide molecule and upon substitution of the halide, the final products would be afforded either (2-a) (H.F= 97.86365, S.E= 44.642), $(11-\mathrm{a})(\mathrm{H} . \mathrm{F}=79.10883, \mathrm{~S} . \mathrm{E}=48.500)$ and $(22-\mathrm{a})(\mathrm{H} . \mathrm{F}=70.38823, \mathrm{~S} . \mathrm{E}=$ 44.895) or (2-b) (H.F = 97.0400, S.E= 12.679), $(11-b)(H . F=78.25746$, S.E $=15.223)$ and $(22-b)(H . F=70.16624$, S.E $=13.938)$.

The analysis of the spectral data support the formation of the actual products (2-a), (11-a) and (22-a) rather than (2-b), (11-b) and (22-b) (the proposed products). Besides, it could be noticed that the (H.F.) increased as the size of the alkyl group (R-X) increase. 


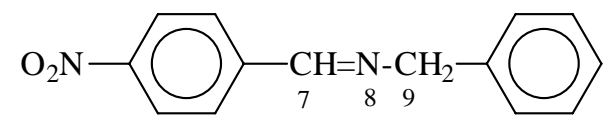

H.F. $=73.40683 \mathrm{Kcal} / \mathrm{mole}$
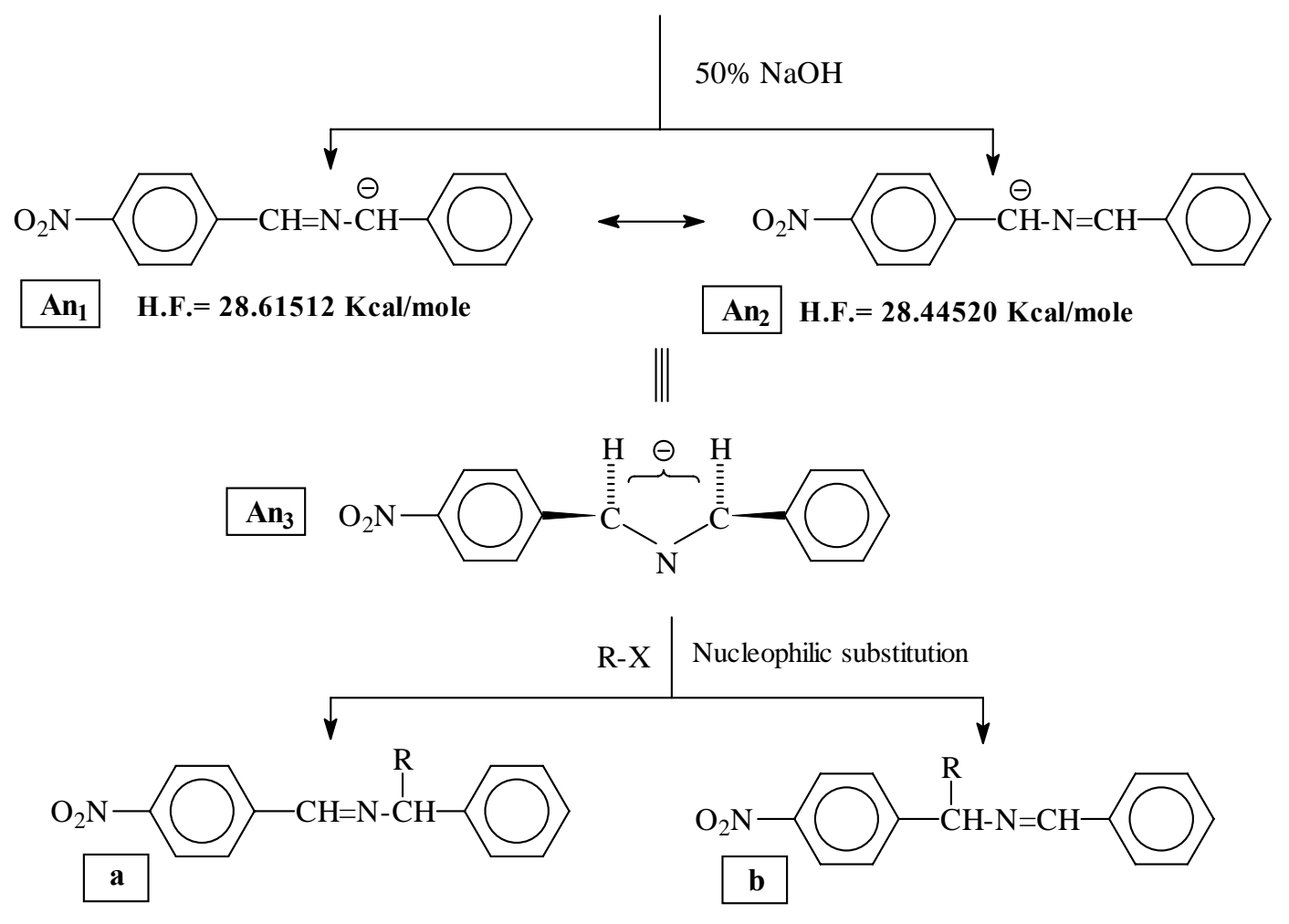

\begin{tabular}{|c|c|c|c|}
\hline Cpd. №. & $\underline{\mathbf{R}}-\underline{\mathrm{X}}$ & H.F.(Kcal/mole $)$ & S.E.(Kcal $/$ mole $)$ \\
\hline $2 a$ & (O)- $-\mathrm{CH}_{2} \mathrm{Cl}$ & 97.86305 & 44.642 \\
\hline $2 b$ & $=$ & 97.04000 & 12.679 \\
\hline $\begin{array}{l}11 a \\
11 b\end{array}$ & $\mathrm{CH}_{3} \mathrm{CH}=\mathrm{CHCH}_{2} \mathrm{Cl}$ & 79.10883 & 48.500 \\
\hline $22 \mathrm{a}$ & $=$ & 78.25746 & 15.223 \\
\hline & $\mathrm{CH}_{3} \mathrm{I}$ & 70.38823 & 44.895 \\
\hline 22b & $=$ & 70.16624 & 13.938 \\
\hline
\end{tabular}

Scheme (2): Reaction of Schiff base(II) with benzyl chloride, crotyl chloride and methyl iodide 
Mowafaq Y. Shandala \& Abdul-Wahab J. Al-Hamdany \& Zaynab W. Majeed \& Omar A. Shareef

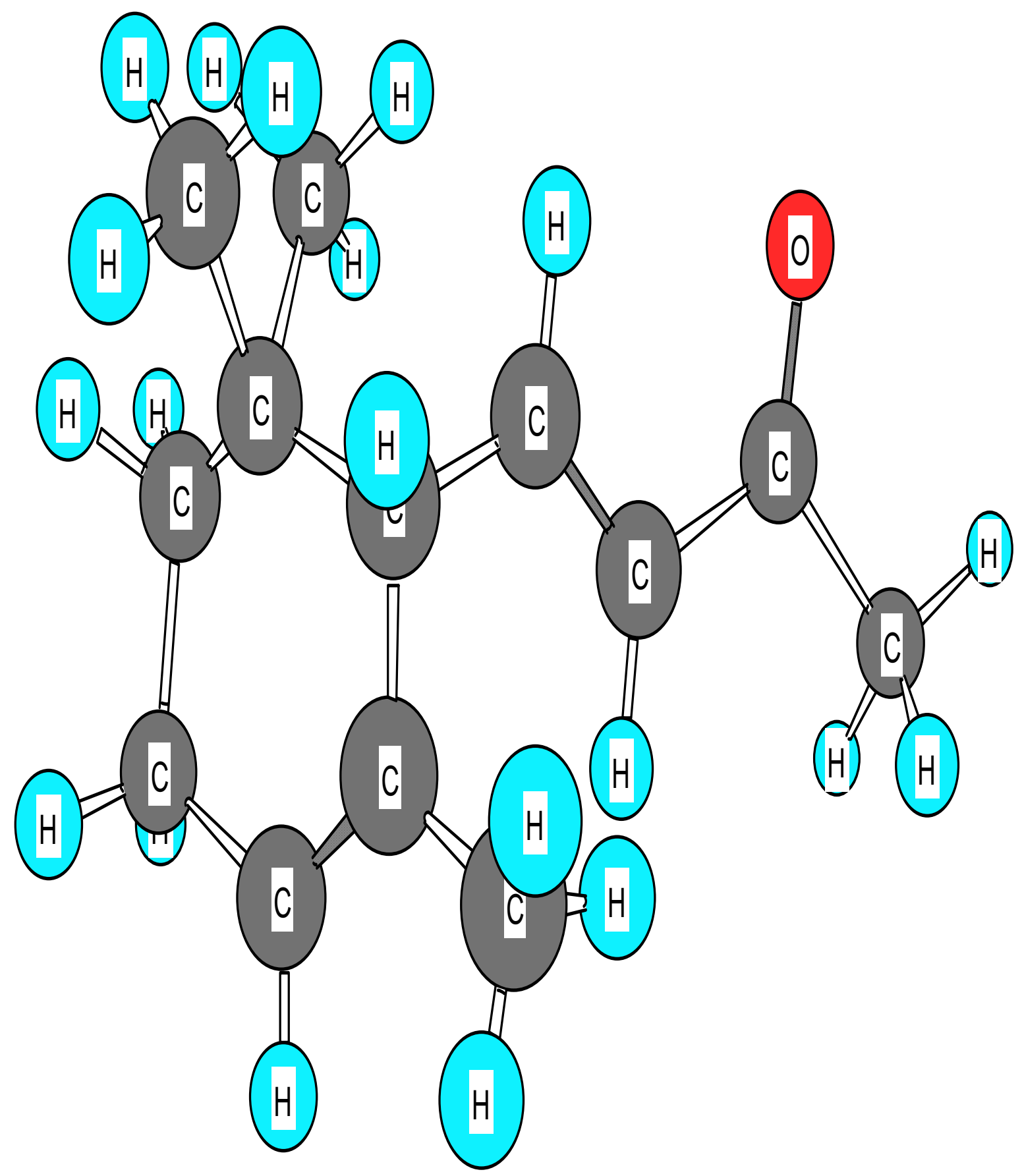

Compound (2-a) Final product

Figure-1- 


\section{Preliminary Biological Study}

In the present work, preliminary biological study is carried out. The biological inhibitory effect (s) of certain products such as: Schiff bases (1, $3,4,28,29)$ against two types of bacterial groups such as: Gram-negative E. coli and Gram-positive Staphylococcus aureus were investigated (table 5).

The isolates were isolated and identified in Biology Dept. in the College of Science in Mosul Univ. The standard Kirby and Bauer ${ }^{(29)}$ method was used.

A loopful of each bacterial species were cultured in nutrient broth and incubated at $\left(37^{\circ} \mathrm{C}\right)$ for $(14-16 \mathrm{hr}$.) and then evently distributed on the nutrient agar by using a sterile swab. The plates were incubated at $\left(37^{\circ} \mathrm{C}\right)$ for (30) min.

The filter paper (Whatmann No.1) discs were distributed on the agar and a certain equal $(1 \mathrm{mg} / 1 \mathrm{ml})$ or $(1 \mathrm{ml} / 1 \mathrm{ml})$ of the compound per solvent (DMSO) was added. The controls here were the Chloramphenicol and / or Ampicillin for comparison. The plates were then incubated at $\left(37^{\circ} \mathrm{C}\right)$ for $(18-24) \mathrm{hr}$. (The interpretation of the results equal the diameter $(\mathrm{mm})$ of the inhibition zone appeared around the discs ${ }^{(30)}$.

The results were interpreted according to the report of the (W.H.O.).

The resistant $(\mathrm{R})$ result represented the diameter of inhibition $<(11)$ $\mathrm{mm}$. However, the moderately sensitive (MS) result was regarded when the zone of inhibition was between (12-16) $\mathrm{mm}$. The sensitive (S) result was over (16) $\mathrm{mm}$.

Table (5): Inhibition effect of certain products on growth of staphylococcus aureus and Escherichia coli

\begin{tabular}{|c|c|c|}
\hline \multirow{2}{*}{ Cpd. No. } & \multicolumn{2}{|c|}{ Test Organism } \\
\cline { 2 - 3 } & Staph. Aureus & E.Coli \\
\hline 1 & S & R \\
\hline 3 & S & MS \\
\hline 4 & S & S \\
\hline 28 & S & S \\
\hline 29 & S & S \\
\hline Chloramphenicol & S & S \\
\hline Ampicillin & S & S \\
\hline
\end{tabular}


Mowafaq Y. Shandala \& Abdul-Wahab J. Al-Hamdany \& Zaynab W. Majeed \& Omar A. Shareef

Appendix (1)

Final Product, Compound (21)

Charges:

\begin{tabular}{|c|c|c|c|}
\hline$C_{1}=-0.141397$ & $C_{2}=-0.115552$ & $C_{3}=-0.139134$ & $C_{4}=-0.112218$ \\
\hline$C_{5}=-0.067222$ & $C_{6}=-0.083705$ & $C_{7}=-0.026542$ & $N_{8}=-0.175310$ \\
\hline$C_{9}=-0.010120$ & $C_{10}=0.069159$ & $C_{11}=0.121431$ & $C_{12}=-0.127911$ \\
\hline$C_{13}=-0.128722$ & $C_{14}=-0.129688$ & $C_{15}=-0.117211$ & $C_{16}=0.235398$ \\
\hline $\mathrm{H}_{17}=\mathbf{0 . 1 3 4 1 8 7}$ & $\mathrm{H}_{18}=\mathbf{0 . 1 3 2 0 6 7}$ & $H_{19}=0.132458$ & $H_{20}=0.130014$ \\
\hline$H_{21}=0.154788$ & $\mathrm{H}_{22}=0.098212$ & $\mathrm{H}_{23}=0.114792$ & $H_{24}=0.129738$ \\
\hline $\mathrm{H}_{25}=\mathbf{0 . 1 3 1 2 7 7}$ & $H_{26}=0.131029$ & $\mathrm{H}_{27}=\mathbf{0 . 1 3 1 4 7 1}$ & $H_{28}=0.133384$ \\
\hline$H_{29}=0.081680$ & $H_{30}=0.088758$ & $H_{31}=0.076863$ & \\
\hline
\end{tabular}

\section{Charges:}

Bond Length $\left(\mathrm{A}^{\circ}\right)$ :

\begin{tabular}{|l|l|l|l|}
\hline $\mathrm{C}_{1} \mathrm{C}_{2}=1.39389$ & $\mathrm{C}_{2} \mathrm{C}_{3}=1.39571$ & $\mathrm{C}_{3} \mathrm{C}_{4}=1.39244$ & $\mathrm{C}_{4} \mathrm{C}_{5}=1.40326$ \\
\hline $\mathrm{C}_{1} \mathrm{C}_{6}=1.39497$ & $\mathrm{C}_{5} \mathrm{C}_{7}=1.47171$ & $\mathrm{C}_{7} \mathrm{~N}_{8}=1.28639$ & $\mathrm{~N}_{8} \mathrm{C}_{9}=1.44964$ \\
\hline $\mathrm{C}_{9} \mathrm{C}_{10}=1.51135$ & $\mathrm{C}_{10} \mathrm{C}_{11}=1.39876$ & $\mathrm{C}_{11} \mathrm{C}_{12}=1.39367$ & $\mathrm{C}_{12} \mathrm{C}_{13}=1.39508$ \\
\hline $\mathrm{C}_{13} \mathrm{C}_{14}=1.39443$ & $\mathrm{C}_{10} \mathrm{C}_{15}=1.39824$ & $\mathrm{C}_{9} \mathrm{C}_{16}=1.53121$ & $\mathrm{C}_{1} \mathrm{H}_{17}=1.10004$ \\
\hline $\mathrm{C}_{2} \mathrm{H}_{18}=1.09983$ & $\mathrm{C}_{3} \mathrm{H}_{19}=1.09978$ & $\mathrm{C}_{4} \mathrm{H}_{20}=1.10049$ & $\mathrm{C}_{6} \mathrm{H}_{21}=1.10139$ \\
\hline $\mathrm{C}_{7} \mathrm{H}_{22}=1.11529$ & $\mathrm{C}_{9} \mathrm{H}_{23}=1.13624$ & $\mathrm{C}_{11} \mathrm{H}_{24}=1.10060$ & $\mathrm{C}_{12} \mathrm{H}_{25}=1.09977$ \\
\hline $\mathrm{C}_{13} \mathrm{H}_{26}=1.09958$ & $\mathrm{C}_{14} \mathrm{H}_{27}=1.09984$ & $\mathrm{C}_{15} \mathrm{H}_{28}=1.10029$ & $\mathrm{C}_{16} \mathrm{H}_{29}=1.11603$ \\
\hline $\mathrm{C}_{16} \mathrm{H}_{30}=1.11616$ & $\mathrm{C}_{16} \mathrm{H}_{31}=1.11630$ & & \\
\hline
\end{tabular}

Bond Angle (degree):

\begin{tabular}{|l|l|l|l|}
\hline$C_{1} C_{2} C_{3}=119.85417$ & $C_{2} C_{3} C_{4}=120.11654$ & $C_{3} C_{4} C_{5}=120.24860$ & $C_{2} C_{1} C_{6}=120.31910$ \\
\hline$C_{4} C_{5} C_{7}=118.26382$ & $C_{5} C_{7} N_{8}=123.19308$ & $C_{7} N_{8} C_{9}=121.72556$ & $N_{8} C_{9} C_{10}=112.21725$ \\
\hline$C_{9} C_{10} C_{11}=120.67568$ & $C_{10} C_{11} C_{12}=120.20796$ & $C_{11} C_{12} C_{13}=120.08087$ & $C_{12} C_{13} C_{14}=119.86615$ \\
\hline$C_{9} C_{10} C_{15}=119.74720$ & $C_{8} C_{9} C_{16}=116.86926$ & $C_{2} C_{1} H_{17}=119.99093$ & $C_{1} C_{2} H_{18}=120.12964$ \\
\hline$C_{2} C_{3} H_{19}=120.00438$ & $C_{3} C_{4} H_{20}=119.77640$ & $C_{1} C_{6} H_{21}=119.75471$ & $C_{5} C_{7} H_{22}=113.14297$ \\
\hline$N_{8} C_{9} H_{23}=103.23022$ & $C_{10} C_{11} H_{24}=119.77215$ & $C_{11} C_{12} H_{25}=119.89536$ & $C_{12} C_{13} H_{26}=120.04793$ \\
\hline$C_{13} C_{14} H_{27}=120.02358$ & $C_{10} C_{15} H_{28}=120.06444$ & $C_{9} C_{16} H_{29}=110.68020$ & $C_{9} C_{16} H_{30}=109.07180$ \\
\hline$C_{9} C_{16} H_{31}=111.14412$ & & & \\
\hline
\end{tabular}

Twist Angle (degree):

\begin{tabular}{|c|c|c|}
\hline $\mathrm{C}_{1} \mathrm{C}_{2} \mathrm{C}_{3} \mathrm{C}_{4}=-\mathbf{0 . 1 0 3 7 4}$ & $\mathrm{C}_{2} \mathrm{C}_{3} \mathrm{C}_{4} \mathrm{C}_{5}=\mathbf{0 . 0 9 4 8 4}$ & $\mathrm{C}_{3} \mathrm{C}_{2} \mathrm{C}_{1} \underline{\mathrm{C}}_{6}=\mathbf{0 . 0 5 3 3 1}$ \\
\hline $\mathrm{C}_{3} \mathrm{C}_{4} \mathrm{C}_{5} \overline{\mathrm{C}}_{7}=-179.75113$ & $\mathrm{C}_{4} \mathrm{C}_{5} \mathrm{C}_{7} \underline{\mathrm{N}}_{8}=-179.22244$ & $\mathrm{C}_{5} \mathrm{C}_{7} \mathrm{~N}_{8} \overline{\mathrm{C}}_{9}=179.28763$ \\
\hline $\mathrm{C}_{7} \mathrm{~N}_{8} \mathrm{C}_{9} \mathrm{C}_{10}=97.57314$ & $\mathrm{~N}_{8} \mathrm{C}_{9} \mathrm{C}_{10} \mathrm{C}_{11}=-62.36017$ & $\mathrm{C}_{9} \mathrm{C}_{10} \mathrm{C}_{11} \mathrm{C}_{12}=-178.97775$ \\
\hline $\mathrm{C}_{10} \mathrm{C}_{11} \mathrm{C}_{12} \mathrm{C}_{13}=\mathbf{- 0 . 0 6 6 5 9}$ & $\mathrm{C}_{11} \mathrm{C}_{12} \mathrm{C}_{13} \mathrm{C}_{14}=-0.19494$ & $\mathrm{~N}_{8} \mathrm{C}_{9} \mathrm{C}_{10} \underline{\mathrm{C}}_{15}=118.19682$ \\
\hline$C_{7} N_{8} C_{9} C_{16}=-28.91081$ & $\mathrm{C}_{3} \mathrm{C}_{2} \mathrm{C}_{1} \mathrm{H}_{17}=-179.96271$ & $\mathrm{C}_{6} \mathrm{C}_{1} \mathrm{C}_{2} \underline{\mathrm{H}}_{18}=-179.95753$ \\
\hline $\mathrm{C}_{1} \mathrm{C}_{2} \mathrm{C}_{3} \mathrm{H}_{19}=179.91753$ & $\mathrm{C}_{2} \mathrm{C}_{3} \mathrm{C}_{4} \overline{\mathrm{H}}_{20}=-179.97464$ & $\mathrm{C}_{2} \mathrm{C}_{3} \mathrm{C}_{4} \overline{\mathrm{H}}_{21}=179.96095$ \\
\hline $\mathrm{C}_{4} \mathrm{C}_{5} \mathrm{C}_{7} \overline{\mathrm{H}}_{22}=7.28229$ & $\mathrm{C}_{7} \mathrm{~N}_{8} \mathrm{C}_{9} \overline{\mathrm{H}}_{23}=-146.20980$ & $\mathrm{C}_{9} \mathrm{C}_{10} \mathrm{C}_{11} \underline{\mathrm{H}}_{24}=1.31820$ \\
\hline $\mathrm{C}_{10} \mathrm{C}_{11} \mathrm{C}_{12} \underline{\mathrm{H}}_{25}=-179.89014$ & $\mathrm{C}_{11} \mathrm{C}_{12} \mathrm{C}_{13} \underline{\mathrm{H}}_{26}=-179.98662$ & $\mathrm{C}_{12} \mathrm{C}_{13} \mathrm{C}_{14} \underline{\mathrm{H}}_{27}=-179.71708$ \\
\hline $\mathrm{C}_{9} \mathrm{C}_{10} \mathrm{C}_{15} \underline{\mathrm{H}}_{28}=-1.38694$ & $\mathrm{~N}_{8} \mathrm{C}_{9} \mathrm{C}_{16} \underline{\mathrm{H}}_{29}=-49.7126$ & $\mathrm{~N}_{8} \mathrm{C}_{9} \mathrm{C}_{16} \underline{\mathrm{H}}_{30}=-169.244$ \\
\hline $\mathrm{N}_{8} \mathrm{C}_{9} \mathrm{C}_{16} \mathrm{H}_{31}=71.44688$ & & \\
\hline
\end{tabular}

Final heat of formation $=66.60970 \mathrm{Kcal} / \mathrm{mole}=278.69797 \mathrm{KJ}$ 


\section{References}

1) C. M. Starks and R. M. Owens, J. Amer. Chem. Soc., 95, 3613-3615, 1973.

2) C. M. Starks, J. Amer. Chem. Soc., 93, 195, 1971.

3) E. V. Dehmlow and S. S. Dehmlow, "Phase Transfer Catalysis", Verlag Chemie., p. 25-36, 1983.

4) M. Solomons, "Fundamentals of Organic Chemistry", $2^{\text {nd }}$ Ed., John Wiley and Sons Inc., New York, p. 349-352, 1986.

5) J. Parekh, P. Inamdhar, R. Nair, S. Baluja, and S. Chanda, J. Serb Chem. Soc.,70(10),1155-1161,2005

6) A. I. Krylov, B. V. Stolyarou, Zh. Org. Khim, 14(1978), 2224.

7) Y. M. Issa, M. M. Omar, H. M. Abdel Fattah, A. A. Soliman, J. Ind. Chem. Soc., 73(1996), 55.

8) S. S. Shetye (Late), M. S. Mayadeo, M. B. Hexameshwar, Asian J. Chem, 11(1999), 1379

9) R. Miao, L. Shouling, Y. Roudong, Y. Lau, Y. Wenbing, Indian J. Chem. Soc. A42(2003)315.

10) B. H. Mehta, V. P. Vengarlekar, Asian J.Chem.,11(1999)397.

11) N. Raman, V. Muthuraj, S. Ravichandranm, A. Kulanndaisamy, Proc. Indian Acad. Soc. 115(2003)161.

12) T. D. Chaudhari, S. S. Subnis, Bull Haskine Inst, 4(1986)85.

13) S. Shah, R. Vyas, R. H. Mehta, J. Indian Chem. Soc., 69(1992)590.

14) N. Raman, A. Kulandaisamy, A. Shunmugasundaram, K. Jeyasubramaniam, Transition Met. Chem.26(2001)131.

15) N. Sari, S. Arslan, E. Logoglu, I. Sakiyan, G. U. J. Sci, 16(2003).283.

16) K. Sahu, R. K.Behera, R. C. Pathaik, A. Nayak, G. B. Behera, Indian J.Chem.188(1979)557.

17) M. Abdul-Gawad, Y. M.Issa, S. M. Abd-Alhamid, Egypt J. Pharm. Sci., 34(1993)219.

18) S. K. Chakraborti, B. Kumar, J. Indian Chem. Soc. LP:137(1973).

19) S. Rao, A. S. Mittra, J. Indian Chem. Soc. LV0420(1978).

20) S. A. Khan, A. A. Siddiqui, S. Bhatt, Asian J.Chem.14(2002).

21) R. L. Shriner, R. C. Fuson, D. Y. Curtin, "The Systematic Identification of Organic Compounds”, $5^{\text {th }}$ Ed., John Wiley \& Sons Inc., New York, p. 29, 1964.

22) R. Chinchilla, N. Galindo, C. Najera, Synthesis, 4, 704-717, 1999.

23) Williams and Fleming, "Spectroscopic Methods in Organic Chemistry", $2^{\text {nd }}$ Ed., McGraw-Hill, England, p. 132, 1973.

24) P. Crews, J. Rodrigues, M. Jaspars, "Organic Structure Analysis", Oxford University Press Inc., p. 62, 1998.

25) Reference 19, p. 18, 29.

26) J. J. Brophy, D. Nelson and J. S. Shannon, Organic Mass Spectrometry, 14, No. 7, p. 379-386, 1979.

27) J. Barker, "Mass Spectrometry", John Wiley \& Sons Ltd., West Sussex, England, p. 28, 1999.

28) E. Ashcroft, "Ionization Methods in Organic Mass Spectrometry", Thomas Graham House, Science Park, Milton Road, Cambridge, U. K., p. 76, 1997.

29) A. W. Bauer, W. M. M. Kirby, J. C. Sherris and M. Turk, Am. J. Clin. Pathol., 45, p. 493-496, 1966.

30) H. M. Ericsson, G. Tunevall and K. Wickman, Scand. J. Clin. Lab. Inves., $\underline{12}$, p. 414-422, 1960. 